\author{
Araştırma Makalesi \\ https://doi.org/10.53803/turvehab.1019837
}

\title{
Türkiye Florası İçin Yeni Bir Kayıt, Muscari commutatum (Asparagaceae)
}

\author{
Tuna Uysal (iD*, Meryem Bozkurt (iD, Hakkı Demirelma
}

Biyoloji Bölümü, Fen Fakültesi, Selçuk Üniversitesi, TR-42130, Konya, Türkiye

"Yazışmadan sorumlu yazar: Tuna Uysal, tuysal@ selcuk.edu.tr

\begin{tabular}{lll}
\hline Geliş: 05.11.2021 & Kabul: 23.11.2021 & Çevrimiçi Yayın: 31.12.2021 \\
\hline
\end{tabular}

\begin{abstract}
Özet
Muscari commutatum türünün varlığı Sicilya (İtalya), Midilli ve Samos adalarından (Ege Denizi) bilinmekteyken, Antalya (Türkiye) ili sınılarından iki farklı lokaliteden toplanmıştır. Bu çalışmada, Türkiye için yeni kayıt olarak belirlenen türün betimi ve yayılışının yanı sıra, SEM aracılığıyla çekilmiş tohum yüzeyi ve karyotip analizine de yer verilmiştir. Morfolojik bulgulara göre, taksonomik açıdan türün en yakın akrabası İsrail ve Ürdün'de doğal olarak yayılış gösteren $M$. filiforme'dir. Tür ilk bakışta perigon yapısı bakımından $M$. latifolium, rengârenk çiçeği ile de $M$. inconstrictum türüyle benzerdir. Türün kromozom sayısı cinsin diğer mensuplarının büyük bir kısmında olduğu gibi $2 n=18$ olup, diploittir. Tohum yüzeyi süsleri ise ağsıdır.
\end{abstract}

Anahtar kelimeler: Flora, Muscari, Rengarenk sümbül, Türkiye, yeni kayıt

\section{Muscari commutatum (Asparagaceae), A New Record for the Flora of Turkey}

\begin{abstract}
While the existence of Muscari commutatum is known from Sicily (Italy), Lesbos and Samos Islands (Aegean Sea), it was collected from two different localities at the borders of Antalya Province (Turkey). In this study, besides the description and distribution of the newly recorded species for Turkey, the seed surface taken by SEM and karyotype analysis were included. According to morphological findings, the taxonomically closest relative of the species is M. filiforme, which naturally occurs in Israel and Jordan. At first glance, M. commutatum appears like M. latifolium for perigonal structure and $M$. inconstrictum with its concolorous flowers. The chromosome number of the species is $2 n=18$, as in most other members of the genus, and it is diploid. Seed surface ornamentations are reticulate.
\end{abstract}

Keywords: Concolorous grape hyacinth, flora, Muscari, new record, Turkey

\section{GíRiş}

Muscari Mill. Asparagaceae familyasına ait soğanlı bir cinstir. Akdeniz havzası, Orta ve Güneybatı Avrupa, Kafkaslar, Güneybatı Asya ve Orta Asya'da geniş bir yayılış alanına sahiptir (Speta 1998; Jafari vd. 2008). Cins, Flora Europaea eserinde 13 (Davis \& Stuart 1980), Flora of the U.S.S.R. eserinde 18 (Lozina-Lozinskaya 1968) ve Flora Iranica eserinde 7 (Rechinger 1990) tür ile temsil edilmektedir. Flora of Turkey and the East Aegean Islands eserinde Muscari cinsinin revizyonu Davis ve Stuart (1984) tarafından gerçekleştirilmiştir. İlgili revizyonda Sicilya, Midilli ve Samos adalarından kaydı verilen Muscari commutatum Guss. dahil 20 türün varlığ 1 bildirilmiştir. Sonradan yapılan çalışmalarda $M$. cammutatum'un Balkanlarda da yetiştiği tespit edilmiştir (Doussi vd. 2002). Bu revizyon sonrası, Eker (2012) tarafindan "Türkiye Bitkileri Listesi (Damarlı Bitkiler)"

Önerilen Alıntı:

Uysal, T., Bozkurt, M. \& Demirelma, H. (2021). Türkiye Florası İçin Yeni Bir Kayıt, Muscari commutatum (Asparagaceae). Türler ve Habitatlar 2(2): 123-133. 
isimli eserde cinsin Türkiye'de yetişen taksonları için bir güncelleme yapılmış ve listede 30 türe yer verilmiştir. Son ylllarda, Türkiye için 15 yeni tür, dört yeni kayıt, iki taksonomik diriltme ve Muscari'den Bellevalia Lapeyr.'ya aktarılan taksonomik bir transfer rapor edilmiştir (Demirci vd. 2013; 2014; Pirhan vd. 2015; Yıldırım 2015; 2016; Çilden \& Yıldırımlı 2017; Pınar vd. 2018; Eker 2019a; 2019b; Demirci Kayıran vd. 2019; Eker vd. 2019; Doğu \& Uysal 2019; Eroğlu vd. 2019; Eroğlu ve Pınar 2019; Yıldırımlı \& Kılıç 2019; Eker vd. 2020; Eker \& Armağan 2020; Eker \& Kandemir 2020, Eker \& Y1ldırım 2021; Eker 2021).

Türkiye Muscari cinsinin revizyonunu amaç edinen TÜBİTAK projesi kapsamında 2019-2020 yıllarında gerçekleştirilen arazi çalışmaları esnasında çok aşina olunmayan ilginç bir Muscari popülasyonuna rastlanmış ve örnekler toplanmıştır. Detaylı morfolojik inceleme ve ilgili literatürler 1şı̆̆ında yapılan değerlendirme sonucunda, toplanan örneklerin Muscari commutatum olduğu belirlenmiştir. Kaya (2014) tarafindan "Türkiye Geofitleri” isimli eserde MU4818-3120 ve MU3203-4806 numaralı iki örneğin $M$. commutatum türüne ait olduğu bildirilmiş ve saksıda canlı çekilmiş resimleri verilmiştir. Yapılan alan çalışmalarıyla Kaya (2014) tarafından bildirilen lokalitelerden örnekler toplanmış ve "Türkiye Geofitleri" isimli eserde verilen resim ve bilgilerle eşleştirilmiştir. Eşleştirme sonucu, ilkinin M. serpentinicum Yıldırım, Altıŏlu \& Pirhan diğerinin ise M. microstomum P.H.Davis \& D.C.Stuart türüne ait olduğu belirlenmiştir. Sonuç olarak, $M$. commutatum türü ilk kez bu çalışma ile Türkiye için yeni bir kayıt olarak rapor edilmiştir. Ayrıca türün meyve yüzey mikromorfolojisi ve karyomorfolojik özellikleri verilmiştir.

Bitki karyotip araştırmaları, bitki türlerinin kökenini ve evrimini, moleküler filogeniyi ve floristik coğrafyayı anlamak için önemlidir (Sun vd. 2019). Karyomorfolojik çalışmalar filogenetik bir basamakta bitki çeşitliliğinin evrimini yönlendiren sitolojik mekanizmaların yanı sıra karyotiplerin potansiyel evrimsel karakteristikleri hakkında bilgi sağlamaktadır. Ayrıca karyomorfolojik çalışmalar, bir türün kromozom sayısı, ploidi düzeyi, karyotip asimetrisi ve karyotip varyasyon katsayısı gibi temel sitolojik parametrelerini tanımlayarak bitki türlerini sınıflandırmak için hızlı ve kolay bir yaklaşımdır (Guerra 2008). Bir türün kromozom sayısı ve karyotipi, onun temel genetik bilgisini yansitabilen kararlı özelliklerdendir. Muscari türlerinin çoğunluğu diploid $(2 n=2 x=18)$ iken, birkaç popülasyon poliploiddir (Stuart 1970; Karlén 1984; Dalgıç 1991; Johnson 1994; Özhatay \& Johnson 1996; Johnson \& Brandham 1997; Demirci Kayıran \& Özhatay 2017; Kıran vd. 2020a; 2020b; Bozkurt 2020).

\section{MATERYAL VE METOT}

$\mathrm{Bu}$ çalışmanın materyalini Antalya'dan toplanan Muscari örnekleri oluşturmaktadır. Toplanan örnekler Türkiye ve komşu floralardan yararlanılarak teşhis edilmiş ve G, NYBG ve W herbaryumlarında bulunan örneklerle karşılaştırılmıştır (Post \& Dinsmore 1933; Lozina-Lozinskaya 1968; Davis \& Stuart 1980; 1984; Stuart 1985; Rechinger 1990). Tohum yüzeyi mikro-morfolojisi ile ilgili detaylı görüntülerin elde edilmesinde taramalı elektron mikroskoptan (SEM) faydalanılmıştır. SEM görüntülemeleri Selçuk Üniversitesi İleri Teknoloji Araştırma ve Uygulama Merkezi'nde gerçekleştirilmiştir.

Karyolojik çalışmalar için, toplanan örneklerin bir kısmına ait soğanlar hasar verilmeden saksılara ekilmiş ve canlılığını koruması sağlanmıştır. Saksılardan çıkarılan soğanlar su içinde yüzen köpüğe yerleştirilerek çimlendirilmiş ve ezme tekniği kullanılarak somatik metafazlarda kromozom sayımları yapılmıştır (Goldblatt \& Johnson 1996). Çimlenen kök uçları, $4^{\circ} \mathrm{C}^{\prime} \mathrm{de} 0,002 \mathrm{M}$ 8-hidroksikinolin ile 8 saat ön işleme tabi tutulduktan sonra, Carnoy fiksatifinde 24 saat düşük 
sıcaklıklarda sabitlenmiştir. Boyama işlemi için Carnoy fiksatifine alınan kök uçları, 1 saat boyunca $5 \mathrm{M}$ hidroklorik asit $(\mathrm{HCl})$ ile hidrolize edilmiştir. Daha sonra, \%45 asetik asit eklenmiş \%1'lik aseto-orsein ile boyanmıştır. Tüm sayımlar için, farklı bireylerden en az beş metafaz plakası incelenmiş ve en iyi metafaz görüntüsü elde edildikten sonra Olympus BX53 mikroskobuna monte edilerek Olympus DP72 dijital kamera ile fotoğraflanmıştır. Fotoğraflanan metafaz görüntülerinden KAMERAM programıyla karyotip ölçümleri gerçekleştirilmiş ve çeşitli simetri indeksleri kullanılarak karyomorfolojileri belirlenmiştir. Kromozomların morfolojilerine göre adlandırılması Levan (1964)'a göre, karyotip ölçüleri ve simetri indeks hesaplamaları ise Zarco (1986) ve Paszko (2006)' ya göre gerçekleştirilmiştir.

\section{SONUÇLAR VE TARTIŞMA}

Muscari commutatum Guss., Fl. Sic. Prodr. 1:426, t. 180 f. 4 (1827) (Şekil 1-3).

Tip. [İtalya]: Sicilya, G.Gussone s.n. (BM!).

Betimleme. Soğan 1,6-2,0 × 1,2-1,8 cm, yumurtamsı, soğancıklı değil; dış tunika kâğıtsı, açık kahverengi, iç tunika zarsı, kremsi beyaz renkli ve üzeri beyaz benekli veya değil. Yapraklar her bir sıkapoz için 4-6 adet, şeritsi veya tersmızraksı, sıkapozdan daha uzun veya boyunca, dik-yükselici veya yere yatık (özellikle kayaların gölgesinde), kanallı, üst yüzeyde beyaz çizgili, kenarda küçük çıkıntılı veya pürüzlü, uçta az çok sivri. Sıkapoz 1-2 adet, 12-20 cm uzunluğunda, çoğunlukla tabanda kavisli ve yüzeysel olarak kırmızımsı beneklere sahip. Salkım sık veya orta sıklıkta, bazen gevşek, darca yumurtamsı-konik, çiçekte 1,8-3,0 × 0,8-1,8 cm, meyvede gevşer. Verimli çiçeklerin sapları 0,8-6,0 mm uzunluğunda, aşağı kıvrık veya düz, kısmen meyvede uzar. Perigon rengârenk, erken çiçeklenmede mavimsi, olgunlaşmaya doğru siyahımsı-mor ila kırmızımsı-mor; tüpler tabandan loba doğru daha koyu, kısır çiçekler soluk mavi ila morumsu; loblar yeşilimsi veya morumsu kırmızımsı. Verimli çiçekler 3,5-4,5 × 1,7-2,5 mm, tüpsü veya dar tersyumurtamsı, belirgin şekilde boğazda sıkışmış ve omuzlu; loblar 0,5-1,0 mm, hafifçe geri kıvrık. Kısır çiçekler 1,2-2,8 × 1,0-2,2 mm, yarı küremsi ila terskonik; saplar 0,2-3,0 mm uzunluğunda, dik. Sitamenler iki sıralı, genellikle sitilus kadar, filamentler $0,3-0,5 \mathrm{~mm}$ uzunluğunda, grimsi mavi, tüpün ortasından veya biraz yukarısından çıkar; anterler $0,7-0,9(-1,0) \times 0,5-0,6 \mathrm{~mm}$, dörtgenimsi, sırttan bağlı, grimsi veya siyahımsı mor; polenler beyazımsı. Ovaryum 1,5-1,6 × 1,5-1,7 mm, yeşil veya grimsi mor, dairesel, üçgensi; sitilus $0,5-0,8(-1,0)$ mm uzunluğunda, narin, beyazımsı gri, başçıklı. Kapsül 7,0-8,0 × 6,0-7,5 mm, üçgensi, terskalpsi kapakçıklı ve genel hatlarıyla dairesel. Tohumlar $0,8-1,2 \times 0,6-1,0 \mathrm{~mm}$, yumurtams1, kese şekilli, siyah, ağsı yüzeyli.

Description. Bulb 1.6-2.0 × 1.2-1.8 cm, ovoid, without bulblets; outer tunics papery, light brown, inner tunics membranous, creamish white having white dots. Leaves $4-6$ per scape, $27-35 \mathrm{~cm}$ long, 2-6 $\mathrm{mm}$ wide, linear to oblanceolate, longer or than scape or throughout, erect-spreading or particularly patent (in canopy of the rocks), canaliculate, with a white median line on upper surface, minutely scabridulous at margin, apex subacute to acute. Scape 1-2, 12-20 cm long, mostly flexouse at the base and superficially having reddish spots. Raceme dense to moderately dense, sometimes lax, narrowly ovoid to conical, $1.8-3.0 \times 0.8-1.8 \mathrm{~cm}$ in flower, accrescent in fruit. Pedicels of fertile flowers $0.8-6.0 \mathrm{~mm}$ long, patent to deflexed, slightly elongate in fruit. Perigon concolorous, bluish in early flowering, blackish-purple to dark magenta towards to maturation; tubes more darker from base to upwards lobes, sterile flowers pale blue to violaceous and lobes greenish or magenta. Fertile flowers 3.5-4.5 × 1.7-2.5 mm, tubular or narrowly obovoid, strongly 
constricted and shouldered; lobes $0.5-1.0 \mathrm{~mm}$, slightly recurved. Sterile flowers $1.2-2.8 \times 1.0-2.2$ $\mathrm{mm}$, semiglobose to obconical; pedicels $0.2-3.0 \mathrm{~mm}$ long, erect. Stamens biseriate, arise usually from style level, filaments $0.3-0.5 \mathrm{~mm}$ long, greyish blue, lower series attached from middle ot slightly upper of tube; anthers oblong, dorsifixed, greyish or blackish purple, $0.7-0.9(-1.0) \times$ 0.5-0.6 mm; pollens whitish. Ovary 1.5-1.6 × 1.5-1.7 mm, green or greyish purple, orbicular, triangular; style 0.5-0.8(-1.0) mm long, slender, whitish grey, capitate. Capsule 7.0-8.0 × 6.0-7.5 $\mathrm{mm}$, trigonous, with obcordate valves and orbicular in outline. Seeds $0.8-1.2 \times 0.6-1.0 \mathrm{~mm}$, ovoid, sack shaped, black, with reticulate surface.

Türkçe isim. Rengârenk Sümbül

Habitat ve ekoloji. Tür genellikle kızılçam ormanı açıklıklarındaki serpantin taşlık-kayalık yamaçlarda (Antalya, Kumluca, Adrasan) veya kalker kaya gölgeliklerinde (Antalya, Kemer, Tahtalı Dağı), 5-750 metre aralığındaki yükseltilerde seyrek olarak dağılış gösterir. Türün çiçeklenme zamanı mart ayı, meyvelenme zamanı nisan ayıdır. Muscari racemosum Mill., Gagea juliae Pascher, Romulea bulbocodium (L.) Sebast. \& Mauri var. bulbocodium, Tulipa orphanidea Boiss. ex Heldr., Dactylorhiza iberica (M.Bieb. ex Willd.) Soó ve Ornithogalum alpigenum Stapf türle aynı habitatı paylaşan diğer geofitlerdir.

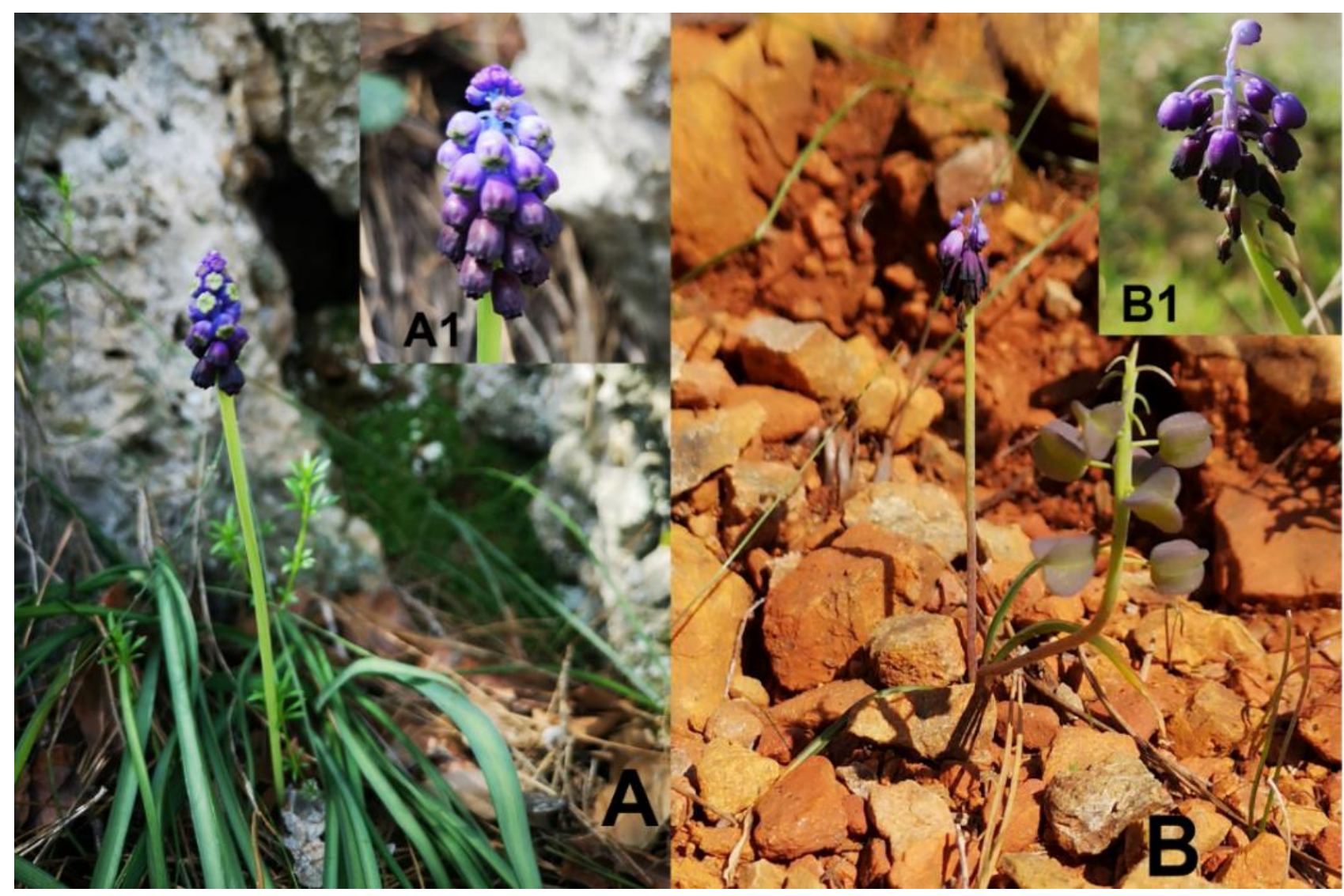

Şekil 1. Muscari commutatum türüne ait Kumluca (A, A1) ve Kemer (B, B1) popülasyonlarının genel ve salkım görünümü.

\section{Taksonomik notlar}

Yeni kayıt olarak tanımlanan türün en yakın akrabası Muscari latifolium J.Kirk türü olarak değerlendirilebilir. İki tür özellikle çiçek rengi ve omurgalı perigon tüpü özellikleriyle benzerdir. Bununla birlikte $M$. commutatum şeritsi-tersmızraksı çok sayıda yaprağa sahip olması ve çiçek 
renginin tüpün tabanından loblara doğru ton değişimi göstermesi ile $M$. latifolium'dan kolayca ayırt edilir. M. commutatum çiçeğindeki rengarenk ton değişimine benzer bir durum $M$. inconstrictum Rech.f. türünde de görülür. Ancak bu türün çiçeklerinin boğaz kısmında ne ciddi bir daralma ne de tüp kısmında omurgalı yapı görülmez. M. commutatum türünün $M$. neglectum Guss. ex Ten. türüne çiçek rengi açısından benzediği, ancak olgun çiçeklerinde lobların siyahımsı-menekşe oluşu ve uca doğru belirginleşen omurgalı yapısı ile ayrıldığı belirtilmiştir (Garbari 1984; Doussi vd. 2002) (Şekil 2).

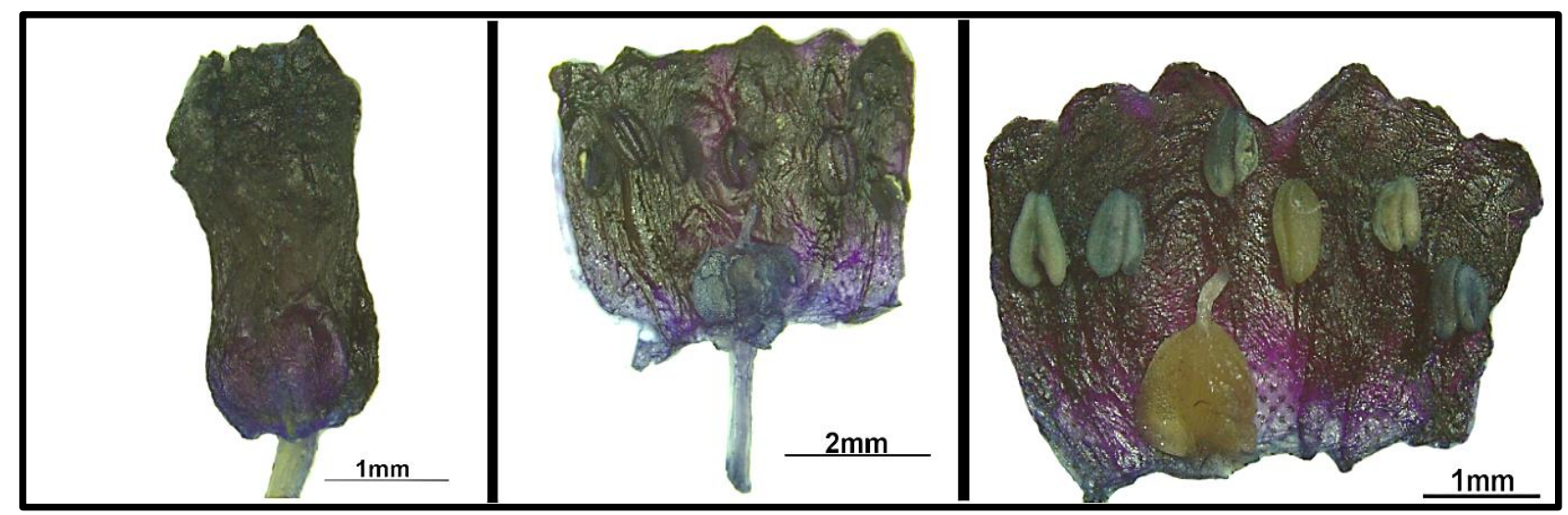

Şekil 2. Verimli çiçek ve iç görünümü.

Türle ilgili tayin çalışmalarına destek sağlaması bakımından morfolojik bulguların yanı sıra karyolojik ve mikromorfolojik çalışmalardan da faydalanılmıştır. Muscari commutatum türünün tohumları yumurtams1, parlak, siyah, 1,36-1,43 × 1,17-1,30 mm olup, tohum süslemesi ağs1 yapıda ve antiklinal ve periklinal hücre duvarı ve epidermal hücre şekli belirsizdir (Şekil 3). Eroğlu vd. (2021) tarafından; $M$. inconstrictum türünün tohumlarının küremsi, 1,99 × 1,80 mm, tohum süslemesinin kısmen ağsı, antiklinal hücre duvarının kabarık, periklinal hücre duvarının çukurlu ve poligonal epidermal hücre şekline sahip olduğu, M. latifolium'un ise tohumlarının yumurtams1küresel, 2,39 × 1,98 mm, antiklinal ve periklinal hücre duvarının ve epidermal hücre şeklinin belirsiz olduğu rapor edilmiştir. Buna göre; $M$. commutatum türünde tohum yüzey yapısının her iki türden de belirgin olarak farklı olduğu görülmektedir.

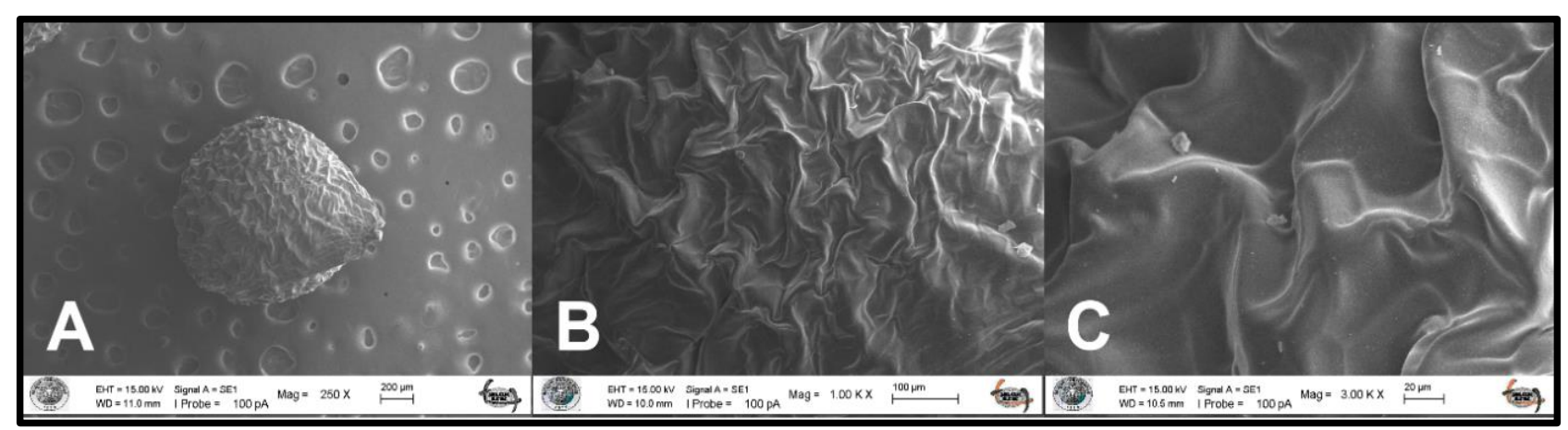

Şekil 3. Muscari commutatum (T. Uysal 3976) türünde tohum yüzeyi SEM görüntüleri (A: 250X, B: $1.00 \mathrm{~K} \mathrm{X}, \mathrm{C}: 3.00 \mathrm{~K} \mathrm{X})$.

Muscari commutatum türüne ait incelenen her iki popülasyonda da kromozom sayısı $2 n=18$ ve karyotip formülü $14 \mathrm{~m}+4 \mathrm{sm}$ olarak belirlenmiştir (Tablo 1, Şekil 4). İntrakromozomal asimetri 
indeksleri $\left(\mathrm{M}_{\mathrm{CA}}\right)$ 15,87 ve 17,61, kromozomlar arasi asimetri indeksleri ise $\left(\mathrm{CV}_{\mathrm{CL}}\right)$ 24,22 ve 25,44'dür (Tablo 2). Kromozomal indislere göre (yüksek asimetri indeksi ve sentromer indeksi varyasyonuna göre) türün kromozomları ileri seviyede farklılaşmış ve evrimleşmiş görülmektedir. M. commutatum türüne ait Delaunay $(1915 ; 1926)$ tarafindan yapılan çalışmalarda kromozom sayısı $2 n=45$ olarak rapor edilmiş, ancak sonraki araştırıcılar tarafından teyit edilmediği için bu bulgu şüpheli olarak kabul edilmiştir (Karlén 1984). Karlén (1984) tarafindan Yunanistan'da yayılış gösteren $M$. commutatum türünün 36 popülasyonu üzerinde karyolojik çalışmalar gerçekleştirilmiş ve tamamında kromozom sayısı $2 n=18$ olarak bildirilmiştir. Bununla birlikte sıra dışı bir biçimde Samos adasından alınan iki popülasyonun üç bireyinde kromozom sayısının $2 n=27$ kromozoma sahip olduğu ve bu triploid bireylerin çiçek boyutunun diploid bireylerden alışılmadık derecede iri olduğu rapor edilmiştir. Kromozom sayımının yanı sıra, Karlén (1984) türün karyotip analizini yapmış ve daha uzun olan birinciden beşinci çift kromozomun belirli bir düzeyde azaldığını ve kromozomların metasentrikten submetasentrik kromozomlara değiştiğini bildirmiş ve kalan dört çift kromozomun ise küçük ve metasenrik olduğunu belirtmiştir. Garbari (1968) tarafindan yapılan çalışmada türün kromozom sayısı $(2 n=18)$ ve morfolojisi hakkında bilgi verilmiştir. Ayrıca Karlén (1984) ve Garbari (1968) tarafından yapılan bu çalışmalarda, ikinci çift kromozomun kısa kolunda satellit olduğu rapor edilmiştir. Antalya'dan toplanan örneklerin karyomorfolojilerinin metasentrik ve submetasentrik kromozomlardan oluşması ve satellitin ikinci kromozomun kısa kolunda yer alması, bu örneklerin Türkiye için yeni kayıt olarak sunulan $M$. commutatum türüne ait olduğunu doğrulamakta ve desteklemektedir (Tablo 1, Şekil 4).

Tablo 1. Muscari commutatum taksonlarına ait populasyonların kromozom özellikleri; DKDeğişim Katsayısı, DO- En Uzun Kromozomun En Kısa Kromozoma Oranı, KKU-Kısa Kol Uzunluğu, UKU-Uzun Kol Uzunluğu, KU-Kromozom Uzunluğu, TKU- Toplam Haploid Kromozom Uzunluğu, Sİ-Sentromerik İndeks, KF- Karyotip Formülü, SS-Standart Sapma: mmetasentrik, sm-submetasentrik.

\begin{tabular}{|l|c|c|c|c|c|c|c|c|c|}
\hline $\begin{array}{l}\text { Koleksiyon } \\
\text { numarası }\end{array}$ & $\mathbf{2 n}$ & $\begin{array}{c}\text { DK } \\
(\boldsymbol{\mu m})\end{array}$ & DO & $\begin{array}{c}\text { KKU } \\
(\boldsymbol{\mu m}) \\
( \pm \mathbf{S D})\end{array}$ & $\begin{array}{c}\text { UKU } \\
(\boldsymbol{\mu m}) \\
( \pm \mathbf{S D})\end{array}$ & $\begin{array}{c}\text { KU } \\
(\boldsymbol{\mu m}) \\
( \pm \mathbf{S D})\end{array}$ & $\begin{array}{c}\text { TKU } \\
(\boldsymbol{\mu m})\end{array}$ & $\begin{array}{c}\text { SI } \\
( \pm \mathbf{S D})\end{array}$ & $\begin{array}{c}\text { KF } \\
\mathbf{S S}\end{array}$ \\
\hline T.Uysal & 18 & $3,24-$ & 2,109 & $\begin{array}{c}2,04 \\
( \pm 0.53)\end{array}$ & $\begin{array}{c}2,81 \\
( \pm 0.73)\end{array}$ & $\begin{array}{c}4,85 \\
( \pm 1,18)\end{array}$ & 43,686 & 42 & $14 \mathrm{~m}+$ \\
\hline T.Uysal & 18 & $2,76-$ & 2,081 & 1,73 & 2,47 & 4,20 & 37,802 & 41 & $14 \mathrm{~m}+$ \\
3976 & & 5,74 & & $( \pm 0,47)$ & $( \pm 0,68)$ & $( \pm 1,07)$ & & $( \pm 0,05)$ & $4 \mathrm{sm}$ \\
\hline
\end{tabular}

Tablo 2. Muscari commutatum taksonlarına ait populasyonların karyotip indeskleri; $\mathrm{A}_{1-}$ İntrakromozomal Asimetri, $\mathrm{A}_{2}$-İnterkromozomal Asimetri, KUVK-Kromozom Uzunluğu Varyasyon Katsayısı, KİVK- Kromozomal İndeks Varyasyon Katsayıs1, Aİ-Asimetri İndeksi, OSAOrtalama Sentromerik Asimetri.

\begin{tabular}{|l|c|c|c|c|c|c|}
\hline Koleksiyon numarası & $\mathbf{A}_{\mathbf{1}}$ & $\mathbf{A}_{\mathbf{2}}$ & KUVK & KíVK & A I & OSA \\
\hline T.Uysal 3970 & 0,259 & 0,242 & 24,228 & 10,556 & 2,557 & 15,87 \\
\hline T.Uysal 3976 & 0,283 & 0,254 & 25,442 & 11,064 & 2.815 & 17,61 \\
\hline
\end{tabular}




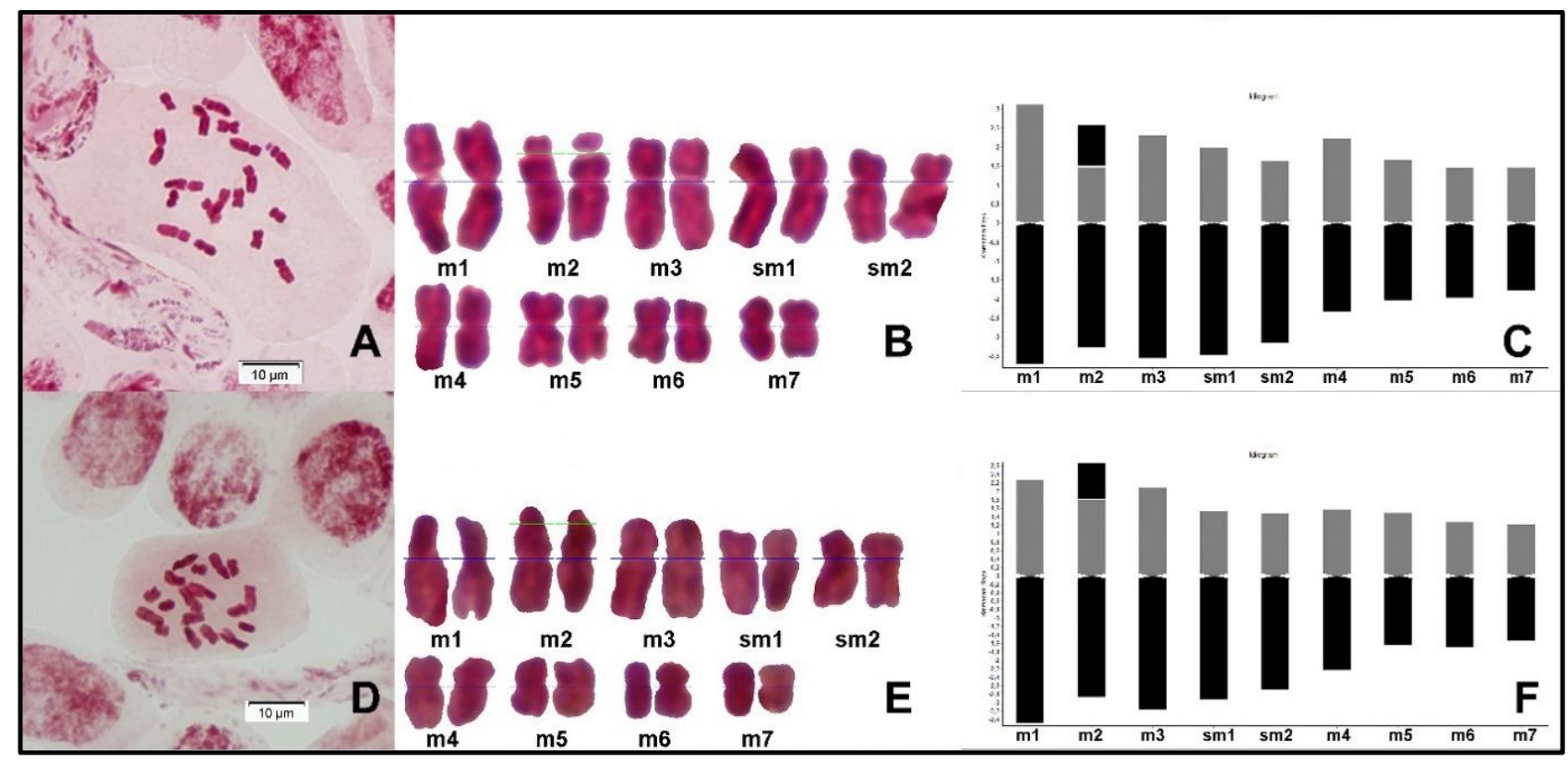

Şekil 4. Muscari commutatum'un Türkiye popülasyonlarına ait metafaz, karyotip ve idiogramları (A-C: Kemer (T.Uysal 3970), D-F: Kumluca (T.Uysal 3976).

\section{İncelenen örnekler}

Muscari commutatum. TÜRKIYE. Antalya: Tahtalı Dağı, teleferik tesisinin kuzeydoğusu, taşlık kayalık sekiler, kaya oyukları, 735-745 m, 24.03.2019, T.Uysal 3808 (KNYA!); Kemer, Tahtalı Dağı teleferik civarı kalker kayalık alan, 720 m, 07.03.2020; T.Uysal 3970 \& H.Demirelma (KNYA!); Kumluca, Adrasan beldesi, denize bakan güney eğimli orman yamaçları ve açıklıkları, 5-60 m, 07.03.2020; T.Uysal 3976 \& H.Demirelma (KNYA!); Kuyucak Dağ1, close to Altıkaya, around and above the remains of ancient Selge, 900-1200 m, 20.04.2006, Staudinger 11743 (W!); İzmir: Efes antik kenti, Bülbül Dağ1, 25-200 m, 25.03.2002, F.Speta s.n. (W!); İTALYA. Italy: Palermo, 04.1893, H.Ross s.n. (NYBG, No: 85'); 1965, D.Stuart s.n. (WU, 0079224!).

Muscari latifolium. TÜRKIYE. Balıkesir: Balıkesir-Edremit, Kaz Dağları, Milli Parkı, Tozluca mevkisi, dere yatağına bakan batı yamaçlar, $1280 \mathrm{~m}, 31.03$. 2018, T.Uysal 3416 \& M.Bozkurt (KNYA!); Edremit (Zeytinli), Kaz Dağları Milli Parkı, Tozlu mevkisi, Orman açıklıkları 1250-1600 m, 28.05.2021; T.Uysal 4332 (KNYA!); Kütahya: Şaphane, Şaphane-Demirören köyü arası, 1480-1550 m, meşe altları, 27.05.2021, T.Uysal 4330 (KNYA!).

Muscari inconstrictum. TÜRKIYE. Hatay: Kırıkhan, Beyazıt-1 Bestami Türbesi civarı, kaya çatlakları, 170 m, 08.04.2018, K.Ertuğrul 5520 (KNYA!); Kırıkhan, Bayezid-i Bestami Türbesi, türbeye çıkışta kayalık seki yarıkları, 15.03.2019, T.Uysal 3757 (KNYA!); Gaziantep: HamidiyeKuzuoluk köyü arası, Kuzuoluk-Kırkpınar köyleri yol ayrımından sola, orman yolundan 500 m, yol kenarı, Pinus açıklıkları, 09.04.2018, K.Ertuğrul 5522 (KNYA!); Hamidiye köyünden KuzuolukKırkpınar köyleri yol ayrımına varmadan $500 \mathrm{~m}$ önce, Pinus açıklıkları, 09.04.2018, K.Ertuğrul 5523 (KNYA!); Yamaçoba köyü, Yamaçoba Barajı kenarı, 1230 m, taşlı yamaçlar, 09.04.2018, K.Ertuğrul 5525 (KNYA!); Sarıkaya köyü dereye bakan kuzey yamaçlar, Asar Tepeler, 1000 m, 16.03.2019, T.Uysal 3760 (KNYA!); Hamidiye- Kuzuluk yolu, Kırkpınar kavşağına gelmeden 500 m, yolun sağındaki döküntülü yamaçlar, Çam açıklıkları, 960-970 m, 16.03.2019, T.Uysal 3764 (KNYA!). 


\section{TEŞEKKÜR}

KBAG-117Z222 numaralı projeyi destekleyen TÜBİTAK'a teşekkür ederiz.

\section{YAZAR KATKI BEYANI}

Bu makalede; çalışma fikri ve tasarımı: Tuna Uysal, veri toplama: Tuna Uysal, Hakkı Demirelma, sonuçların analizi ve yorumlanması: Tuna Uysal, Meryem Bozkurt, Hakkı Demirelma, makale taslağının yazımı: Tuna Uysal, Meryem Bozkurt tarafından yapılmıştır. Yazarlar sonuçları gözden geçirmiş, baskı öncesi makalenin son halini kontrol etmiş ve onaylamıştır.

\section{KAYNAKLAR}

Bozkurt, M. (2020). Karyological Analysis of Endemic Five Muscari Taxa. Cytologia 85(4): 289294. DOI: https://doi.org/10.1508/cytologia.85.289.

Çilden, E. \& Yıldırımlı, Ş. (2017). Reseda anatolica, a new status for Reseda inodora var. anatolica (Resedaceae), and Bellevalia sirnakense (Hyacinthaceae), a new combination from Turkey. Ot Sistematik Botanik Dergisi 24: 37-44.

Dalgıç, G. (1991). Cytotaxonomic studies on the genus Muscari in European Turkey. Botanika Chronika 10: 819-825.

Davis, P.H. \& Stuart, D.C. (1980). [Muscari Mill.] In: Tutin, T.G., Heywood, V.H., Burges, N.A., Moore, D.M., Valentine, D.H., Walters, S.M. \& Webb, D.A. (Eds.). Flora Europaea. Vol. 5. Cambridge University Press, London, pp. 46-49.

Davis, P.H. \& Stuart, D.C. (1984). [Muscari Mill.] In: Davis, P.H. (Ed.). Flora of Turkey and the East Aegean Islands. Vol. 8. Edinburgh University Press, Edinburgh, pp. 227-263.

Delaunay, L.N. (1915). Etude comparee caryologique de quelques especes du genre Muscari Mill. Mem. Soc. Natural. Kiew (Russian) 25: 33-62.

Delaunay, L.N. (1926). Phylogenetische Chromosomenverkurzung. Zeitschrift Für Zellforschung Und Mikroskopische Anatomie 4: 338-364.

Demirci Kayıran, S. \& Özhatay, F.N. (2017). A karyomorphological study on the genus Muscari Mill. growing in Kahramanmaraş (Turkey). Turk J Bot 41(3): 289-298. DOI: 10.3906/bot1605-6.

Demirci Kayıran, S., Özhatay, N. \& Kaya, E. (2019). Muscari tauricum (Asparagaceae, Scilloideae), a new species from Turkey. Phytotaxa 399(2): 109-118. DOI: https://doi.org/ 10.11646/phytotaxa.399.2.1.

Demirci, S., Özhatay, N. \& Koçyiğit, M. (2013). Muscari erdalii (Asparagaceae, Scilloideae), a new species from Southern Turkey. Phytotaxa 154(1): 38-46. DOI: https://doi.org/10.11646/ phytotaxa.154.1.2.

Demirci, S., Özhatay, N., Gürdal, B. \& Kaya, E. (2014). [Türkiye Geofit Florasına Katkılar (Contributions to the Turkish Geophyte Flora)] In: Kaya, E. (Ed.) Türkiye Geofitleri. Vol. 3. Furkan Ofset, Yalova, pp. 543-552.

Doğu, S. \& Uysal, T. (2019). Muscari savranii (Asparagaceae), a new species from Central Anatolia, Turkey. Phytotaxa 402(3): 155-164. DOI: https://doi.org/10.11646/phytotaxa .402.3.3.

Doussi, M.A. \& Thanos, C.A. (2002). Ecophysiology of seed germination in Mediterranean geophytes. 1. Muscari spp. Seed Science Research 12(3): 193-201. DOI: https://doi.org/ 10.1079/SSR2002111. 
Eker, İ. \& Armağan, M. (2020). Türkiye Florası'nda Muscari kerkis (Asparagaceae)'in varlığı üzerine (On the presence of Muscari kerkis (Asparagaceae) in the Flora of Turkey). Bağbahçe Bilim Dergisi 7(3): 25-34. DOI: 10.35163/bagbahce.764281.

Eker, İ. \& Kandemir, A. (2020). Muscari sintenisii Freyn (Asparagaceae)'nin taksonomik dirilişi ve türün lektotipifikasyonu. Bağbahçe Bilim Dergisi 7(3): 12-24. DOI: 10.35163/bagbahce .769591 .

Eker, İ. \& Yıldırım, H. (2021). Muscari inundatum (Asparagaceae, Scilloideae), a new species from southern Anatolia. Phytotaxa 484(2): 181-194. DOI: https://doi.org/10.11646/phytotaxa .484.2.3.

Eker, İ. (2012). [Muscari Mill.] In: Güner, A., Aslan, S., Ekim, T., Vural, M. \& Babaç, M.T. (Eds.). Türkiye Bitkileri Listesi (Damarlı Bitkiler). Nezahat Gökyiğit Botanik Bahçesi \& Flora Araştırmaları Derneği Yayını, İstanbul, pp. 98-100.

Eker, İ. (2019a). Muscari fatmacereniae (Asparagaceae, Scilloideae), a new species from southern Anatolia. Phytotaxa 397(1): 99-106. DOI: https://doi.org/10.11646/phytotaxa.397.1.10.

Eker, İ. (2019b). Muscari pamiryigidii (Asparagaceae, Scilloideae), a new species from northwestern Anatolia. Phytotaxa 408(4): 255-266. DOI: https://doi.org/10.11646/phytotaxa .408.4.2.

Eker, İ. (2021). What is Muscari massayanum and what is not? Second species born of confusion: Muscari erzincanicum (Asparagaceae, Scilloideae), a new species from Turkey. Phytotaxa 487(1): 041-055. DOI: https://doi.org/10.11646/phytotaxa.487.1.3.

Eker, İ., Yıldırım, H. \& Armağan, M. (2019). Türkiye Florası için yeni bir müşkürüm kaydı: Muscari pallens (A new grape hyacinth record for the Flora of Turkey: Muscari pallens). Bağbahçe Bilim Dergisi 6(1): 45-53. DOI: 10.35163/bagbahce.559583.

Eroğlu, H. \& Pınar, S.M. (2019). The taxonomic resurrection of Muscari haradjianii (Asparagaceae, Scilloideae), and a new synonym in the genus Muscari in Turkey. Phytotaxa 418(1): 097-106. DOI: https://doi.org/10.11646/phytotaxa.418.1.6.

Eroğlu, H., Pınar, S.M. \& Fidan, M. (2019). Muscari sabihapinari sp. nov. (Asparagaceae) from Anatolia, Turkey. Nord J Bot 37(11). DOI: https://doi.org/10.1111/njb.02514.

Eroğlu, H., Karaismailoğlu, M.C., Pınar S.M. \& Fidan, M. (2021). Seed micromorphology and anatomy of 36 Muscari (Asparagaceae) taxa from Turkey with notes on their systematic importance. Acta Botanica Croatica 80(2): 146-157. DOI: https://doi.org/10.37427/botcro2021-015.

Garbari, F. (1968). Il Genere Muscari (Liliaceae): Contributo Alla Revisione Citotassonomica, Giornale Botanico Italiano 102(2): 87-105. DOI: https://doi.org/10.1080/11263506809426 442.

Garbari, F. (1984). Some karyological and taxonomic remarks on the Italian Muscari (Liliaceae). Webbia 38(1): 139-164. DOI: https://doi.org/10.1080/00837792.1984.10670304.

Goldblatt, P. \& Johnson, D.E. (1996). Index to Plant Chromosome Numbers 1992-1993. Vol. 58. Missouri Botanical Garden Press, Missouri.

Guerra, M. (2008). Chromosome numbers in plant cytotaxonomy: concepts and implications. Cytogenetic and Genome Research 120(3-4): 339-350. DOI: https://doi.org/10.1159/ 000121083. 
Jafari, A., Maassoumi, A.A. \& Farsi, M. (2008). Karyological study on Bellevalia and Muscari (Liliaceae) species of Iran. Asian Journal of Plant Science 7(1): 50-59. DOI: 10.3923/ ajps.2008.50.59.

Johnson, M.A.T. (1994). Cytology of three new geophytes from Turkey. Kew Bulletin 49(3): 491498. DOI: https://doi.org/10.2307/4114473.

Johnson, M.A.T. \& Brandham, P.E. (1997). New chromosome numbers in petaloid monocotyledons and in other miscellaneous angiosperms. Kew Bulletin 52(1): 121-138. DOI: https://doi.org/ $10.2307 / 4117845$.

Karlén, T. (1984). Karyotypes and chromosome numbers of five species of Muscari (Liliaceae). Willdenowia 14(2): 313-320.

Kaya, E. (2014). [Muscari commutatum Guss.] In: Kaya, E. (Ed.) Türkiye Geofitleri: Vol. 2. Furkan Ofset, Yalova, pp. 376-377.

Kıran, Y., Pınar, S.M., Dogan, G. \& Eroğlu, H. (2020a). A Karyomorphological Study on the Subgenus Leopoldia of the Genus Muscari Growing in Turkey. Cytologia 85(1): 79-83. DOI: https://doi.org/10.1508/cytologia.85.79.

Kıran, Y., Pınar, S.M., Dogan, G. \& Eroğlu, H. (2020b). A Karyomorphological Study on the Genus Muscari Miller Growing in Turkey. Cytologia 85(4): 301-305. DOI: https://doi.org/ 10.1508/cytologia.85.301.

Levan, A., Fredga, K. \& Sandberg, A.A. (1964). Nomenclature for centromeric position on chromosomes. Hereditas 52(2): 201-220. DOI: https://doi.org/10.1111/j.1601-5223.1964 .tb01953.x

Lozina-Lozinskaya, A.S. (1968). [Muscari Mill.] In: Komarov, V.L. (Ed.). Flora of the U.S.S.R. Vol. 4. Israel Program for Scientific Translations, Jerusalem, pp. 316-324.

Özhatay, N. \& Johnson, M. (1996). Some karyological remarks on Turkish Allium sect. Allium, Bellevalia, Muscari, Ornithogalum subg. Ornithogalum. Bocconea 5(1): 239-249.

Paszko, B. (2006). A critical review and a new proposal of karyotype asymmetry indices. Pl Syst Evol 258(1/2): 39-48. DOI: 10.1007/s00606-005-0389-2.

Pınar, M.P., Fidan, M. \& Eroğlu, H. (2018). Muscari botryoides (L.) Mill.: A new record for the family Asparagaceae from Turkey. Türkiye Tarımsal Araştırmalar Dergisi 5(2): 116-119. DOI: https://doi.org/10.19159/tutad.368374.

Pirhan, A.F., Yıldırım, H. \& Altığlu, Y. (2015). Muscari serpentinicum sp. nova (Asparagaceae): a new species from western Anatolia, Turkey. Ot Sistematik Botanik Dergisi 21: 1-14.

Rechinger, K. (1990). [Liliaceae II.] In: Browicz, K.H., Persson, K. \& Wendelbo, P. (Eds.). Flora Iranica. Vol. 165. Akademische Druck- u. Verlagsanstalt, Graz, pp. 140-148.

Zarco, C.R. (1986). A new method for estimating karyotype asymmetry. Taxon 35(3): 526-530. DOI: https://doi.org/10.2307/1221906.

Speta, F. (1998). [Hyacithaceae] In: Kubitzki, K. (Ed.). The Families and Genera of Vascular Plants. Vol. 3. Springer-Verlag, Heidelberg, pp. 261-285.

Stuart, D.C. (1970). Chromosome numbers in the genus Muscari. Notes Roy Bot Gard Edinburgh 30: 189-196.

Stuart, D.C. (1985). [Muscari Mill.] In: Townsend, C.C. \& Guest, E. (Eds.). Flora of Iraq. Vol 8. Ministry of Agriculture and Agrarian Reform, Baghdad, pp. 126-135. 
Sun, W.G., Sun, H. \& Li, Z.M. (2019). Chromosome data mining and its application in plant diversity research. Plant Science Journal 37(2): 260-269. DOI: 10.11913/PSJ.20950837.2019.20260.

Yıldırım, H. (2015). Muscari atillae sp. nova (Asparagaceae): a new species from eastern Anatolia, Turkey. Phytotaxa 213(3): 291-295. DOI: 10.11646/phytotaxa.213.3.9.

Y1ldirım, H. (2016). Muscari elmasii sp. nova (Asparagaceae): a new species from western Anatolia, Turkey. Turk J Bot 40(4): 380-387. DOI: 10.3906/bot-1507-17.

Yıldırımlı, Ş. \& Kılıç, Ö. (2019). A new species of Muscari Mill. (Asparagaceae/Liliaceae), M. nazimiyensis from Tunceli, Turkey. Ot Sistematik Botanik Dergisi 26: 13-16. 